\title{
EXPERIMENTAL EVALUATION OF CARTESIAN AND JOINT IMPEDANCE CONTROL WITH ADAPTIVE FRICTION COMPENSATION FOR THE DEXTEROUS ROBOT HAND DLR-HIT II
}

\author{
ZHAOPENG CHEN ${ }^{*, \dagger, \ddagger ~ N E A L ~ Y . ~ L I I ~}{ }^{\dagger, \S}$ THOMAS WIMBÖCK $^{\dagger, \uparrow}$, \\ SHAOWEI FAN $*, \|$ and HONG LIU ${ }^{*, \dagger, * *}$ \\ *State Key Laboratory of Robotics and System, \\ Harbin Institute of Technology, \\ 150001 Harbin, China \\ ${ }^{\dagger}$ Institute of Robotics and Mechatronics, German Aerospace Center, \\ 82234 Wessling, Germany \\ ${ }^{\ddagger}$ zhaopeng.chen@dlr.de \\ §neal.lii@dlr.de \\ Thomas.wimboeck@dlr.de \\ "fansw@hit.edu.cn \\ **hong.liu@hit.edu.cn
}

Received 14 April 2011

Accepted 7 October 2011

\begin{abstract}
This paper presents impedance controllers with adaptive friction compensation for the five-finger dexterous robot hand DLR-HIT II in both joint and Cartesian space. An FPGA-based control hardware and software architecture with real-time communication is designed to fulfill the requirements of the impedance controller. Modeling of the robot finger with flexible joints and mechanical couplings in the differential gear-box are described in this paper. In order to address the friction due to the complex transmission system and joint coupling, an adaptive model-based friction estimation method is carried out with an extended Kalman filter. The performance of the impedance controller with both adaptive and parameter-fixed friction compensations for the robot hand DLR-HIT II are analyzed and compared in this paper. Furthermore, gravity estimation is implemented with Least Squares technique to address uncertainties in gravity compensation due to the close proximity and complexity of robot hand components. Experimental results prove that accurate position tracking and stable torque/force response can be achieved with the proposed impedance controller with friction compensation on fivefinger dexterous robot hand DLR-HIT II.
\end{abstract}

Keywords: Dexterous robot hand; Cartesian space; impedance control; adaptive friction compensation.

\section{Introduction}

Researchers have made numerous attempts at duplicating the form and function of the human hand. Numerous multifingered robot hands have been developed mainly 
in two categories: with actuators mounted in the robot hand, or located outside the robot hand, with power transmitted though tendon cables. The latter type of robot hands (e.g., Stanford-JPL hand by Salisbury et al., ${ }^{1}$ the Utah/MIT hand by Jacobsen et al., ${ }^{2}$ and the robonaut hand by Lovchik et al. ${ }^{3}$ ) suffer from the elasticity of the tendon cable due to the inaccurate joint angle control. They are also difficult to realize as commercial products due to their mechanical complexity. However, the former group of robot hands (e.g., the Gifu Hand by Kawasaki et al. ${ }^{4}$ the DLR Hand II by Butterfass, ${ }^{5}$ and the DLR/HIT Hand I by Liu ${ }^{6}$ ) face other problems, such as insufficient fingers and joints, inappropriate large size and weight for practical tasks.

An essential feature of a dexterous robot hand is the capability to keep hold of manipulated objects despite disturbances, while maintaining compliance between the robot finger and the object to avoid damage to the hand and object. Hogan introduced a framework for impedance control, ${ }^{7}$ which can be used to achieve compliant manipulation and reliable grasping with different objects in unknown environments. Impedance control on robot manipulators has been addressed by numerous literatures. An internal force-based impedance control scheme for cooperating manipulators was introduced by Bonitz and Hisa. ${ }^{8}$ Asymptotic stability was proven using Lyapunov theory, and simulation results were presented to validate the proposed concepts. Schneider and Cannon developed an object impedance controller for cooperative manipulation, with experimental results from a dual two-link-arm robotic system demonstrating good performance for the proposed control strategy. ${ }^{9}$ Robot arms, instead of hands, are considered in most of the impedance control literatures. Biagiotti designed an admittance controller for the dexterous robot hand DLR II, ${ }^{10}$ with a local proportional regulator in joint space to deal with singularity conditions of the kinematic structure. Wei et al. developed an impedance controller for multifinger dexterous robot hand DLR-HIT I together with the hardware and software architecture designs. ${ }^{11}$ However, the above works are dependent on six-dimensional force/torque sensors, which suffer from higher level of signal noise than the joint torque sensor-based robotic hands.

Joint flexibility and nonlinear friction are nonnegligible in robot manipulators powered by $\mathrm{DC}$ or $\mathrm{AC}$ motor in series with harmonic drives, which are common in many precision positioning and light-weight applications. ${ }^{12}$ With more compact and anthropomorphic design for robot hands than arms, friction issue arises and dominates the performance of impedance controller.

In order to address high friction in robot arm with impedance control, experimental results are carried out with Stribeck friction model for robot arm by Liu et $a l .{ }^{13}$ Le Tien et al. propose an adaptive impedance controller with friction compensation based on velocity failure for DLR medical robot arms. ${ }^{14}$ However, in the above works, the friction model and system parameters have to be identified or tuned manually.

Singular perturbation model was utilized by Spong et al. to establish an integral manifold approach of feedback control for flexible joint robots. ${ }^{15}$ Another 
decoupling-based method with full state linearization was proposed by De Luca and Lucibello. ${ }^{16}$ Albu-Schaeffer and Ott et al. conducted several experimental investigations on Cartesian impedance control for DLR LWR arm with flexible joints and demonstrated the effectiveness of the proposed control scheme practically. ${ }^{17,18}$ Asymptotic stability has been proven based on the passivity theory.

However, the above works consider joint flexibility on robot arms. Few analysis literature is currently available for modeling and impedance controller implementation for the elastic joints on robot fingers with smaller sizes and more compact design.

In order to achieve anthropomorphic shape and dexterous manipulation ability, the DLR-HIT II hand is designed with a complex transmission system, joint coupling and highly compact mechatronic structures, which cause joint flexibilities and complex friction effects that hinder the compliance control performance. Elastic and coupled joints on robot fingers are modeled similar to robot arms. Joint and Cartesian impedance controllers based on joint torque feedback are implemented in this paper to achieve compliance behavior of robot fingers. An adaptive friction observer based on extended Kalman filter is proposed this paper, which employs a simple friction model and estimates the model parameters to address diverse frictions adaptively for different joints in DLR-HIT II robot hand. On the other hand, the uncertainties of gravity for a single joint are introduced by the close proximity and complex design. A practical gravity compensation method is carried out with Least Squares technique in this paper. Furthermore, an FPGA-based multisensory hardware architecture with real-time communication system is proposed to fulfill the requirements of the designed impedance controller and compact mechanical design.

\section{Dexterous Robot Hand System and Modeling}

\subsection{Hardware and software architecture of DLR-HIT II robot hand}

All five fingers of the dexterous robot hand are of modular design, with identical electromechanical structure and control architecture. As illustrated in Fig. 1, the proposed control architecture consists of multisensory system, finger controller, palm controller, and external real-time controller. For each finger, two custom-designed potentiometers in the proximal joint and a contactless magnetic angle sensor in the finger distal unit are equipped to measure the absolute angular position of the three joints. To precisely measure the external torque without any interference between the two DOFs, a new type of proximal joint torque sensor with two DOFs and a distal torque sensor, both based on strain gauge theory, are adopted for the three joints.

For the proximal joint, the motor driver, communication system, and sensor data acquiring are implemented in one single electronic board in Very-High-Speed Integrated Circuit Hardware Description Language (VHDL) in an FPGA controller, which is physically located at the bottom of the finger base. Two brushless DC 

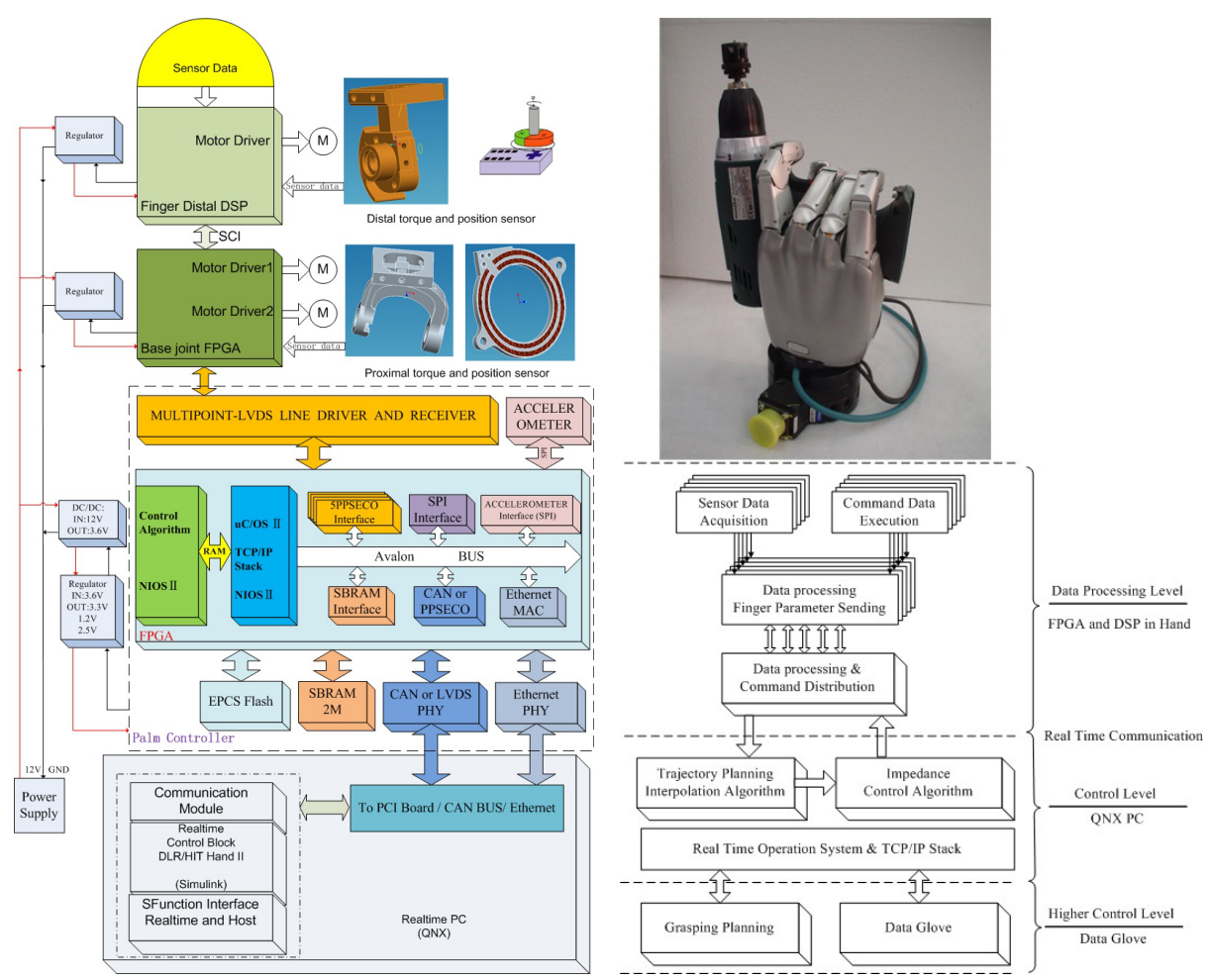

Fig. 1. Hardware and software architectures of the dexterous robot hand DLR-HIT II.

motors are directly driven and controlled by the FPGA together with MOSFET driver gates. In order to achieve modularity of the robot finger, the parameters related to the finger control are stored in the flash memory on the finger base board. The finger distal motor is controlled by a DSP, which is attached on the back side of the first linkage of the finger. A floating-point digital signal processor (DSP) is implemented in the finger distal joint to carry out the sensor signals processing and BLDC motor control, as well as the communication between the finger distal and the finger base. For the palm controller, a Cyclone III FPGA is chosen with NIOS II dual-processors system implemented in a single chip. With its sufficient processing power, a flexible communication system can be achieved in a smaller electronics package to fulfill the hardware real-time requirement. The Cartesian impedance control algorithm, trajectory planning, and dynamic compensation are carried out in a QNX PC with a MATLAB/Simulink-QNX tool chain, as shown in Fig. 1. Motor control data, sensor data, and tracking data are transmitted among finger controller, palm controller, and QNX PC in real time. In order to facilitate the feedback control of the robot hand, a high-speed data bus of M-LVDS is implemented within the FPGA, among the finger controller, palm controller, and QNX PC to realize a communication cycle time of $200 \mu \mathrm{s}$. 


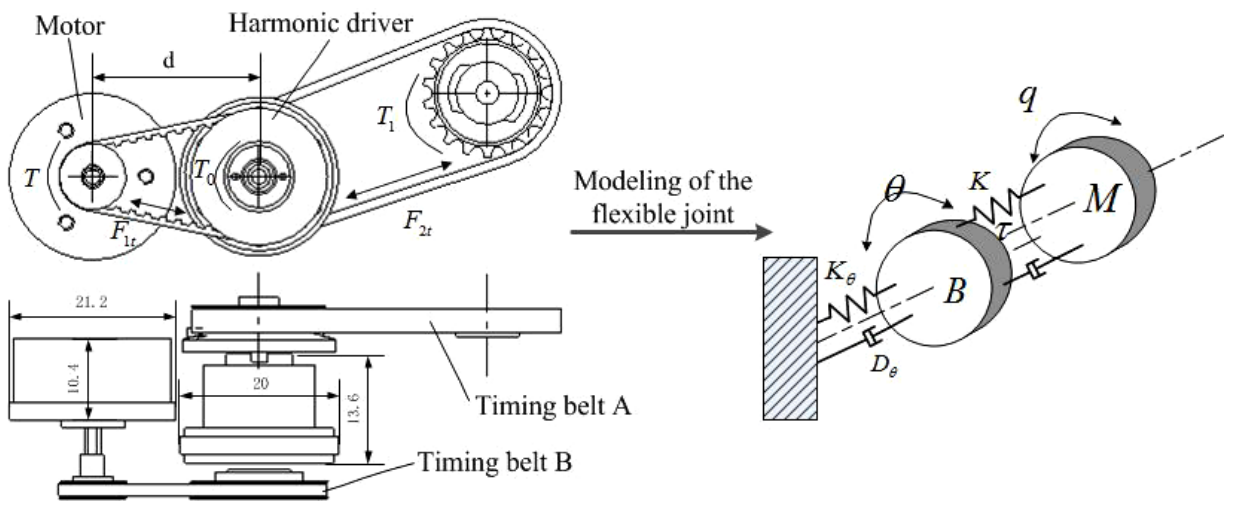

Fig. 2. Transmission system for a single joint in DLR-HIT II robot hand.

\subsection{Modeling with elastic joint and joint coupling}

Experimental results have shown that joint elasticity should be taken into account in the modeling of robotic manipulators. The elastic deformation of the joints, either through deformation of the bearing or of the gear teeth, introduces joint flexibility that has greater influence on control system design than the bending modes of the links, which can be of significantly higher frequency than the resonant flexible modes of the joints. ${ }^{19}$ Each finger of the dexterous robot hand DLRHIT II is driven by three BLDC motors, in series with harmonic drive gears (gear ratio $1: 100$ ) and timing belts (ratio $1: 1.125$ and $1: 2.083$ ). Inherent flexibility of the joint exists in the harmonic drive gear, timing belts, and the torque sensor, which is modeled as a linear torsional spring with stiffness $\boldsymbol{k}$, as shown in Fig. 2. Taking $\tau_{m}$ as the control variable $u$, the single flexible joint dynamics are expressed as ${ }^{15}$ :

$$
\begin{array}{r}
I_{l} \ddot{\vartheta}_{l}+b_{l} \dot{\vartheta}_{l}+g_{l}=k\left(\vartheta_{l}-\vartheta_{m}\right)+z_{e x t}, \\
I_{m} \ddot{\vartheta}_{m}+b_{m} \dot{\vartheta}_{m}+k\left(\vartheta_{l}-\vartheta_{m}\right)=u,
\end{array}
$$

where $\vartheta_{l}$ and $\vartheta_{m}$ are the link side position and motor position, respectively, divided by gear ratio $1 / n$ for a single flexible joint. $n$ represents the transmission ratio. $z_{\text {ext }}$ denotes external torque on link side. $I_{m}$ and $I_{l}$ indicate inertiae of the motor and link, respectively.

The base joint of the robot finger is realized in the form of a coupled joint with differential gears. The joint is powered with two motors, each actuating through a harmonic drive and timing belt assembly. A schematic view for the coupling gears is shown in Fig. 3. The differential gear box consists of four conical gears, allowing the torque of both motors to be directed in either the proximal or abduct direction of motion. This increases the available torque for a given robot size and reduces the total mass of the design. Without considering elasticity, the transformations of 


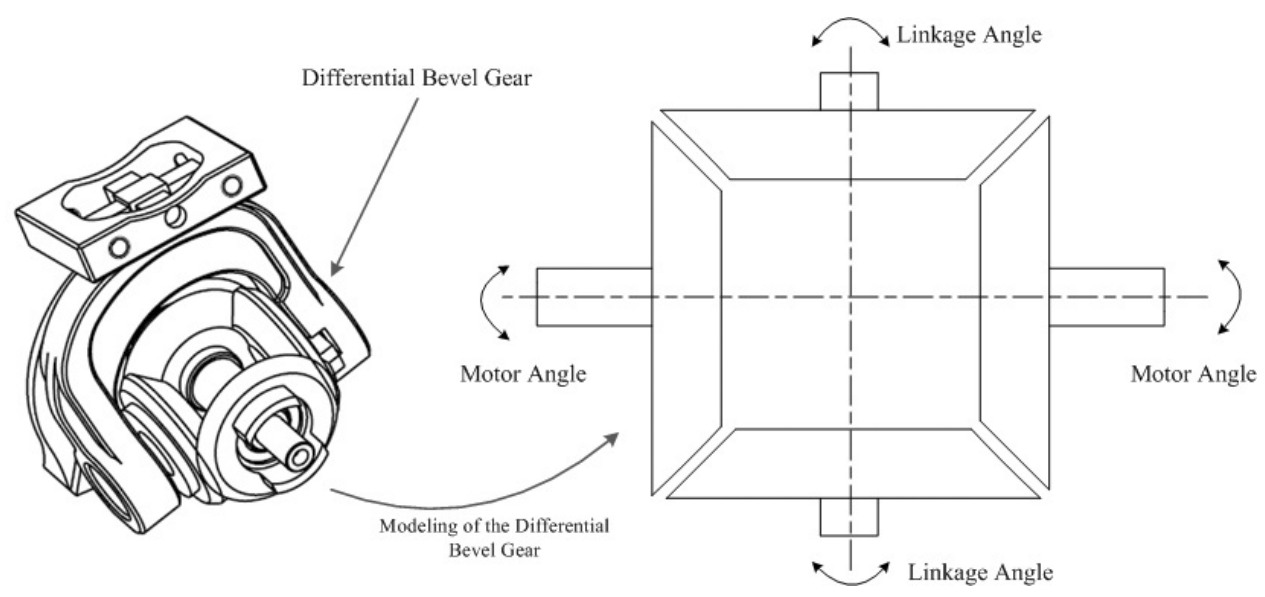

Fig. 3. Differential bevel gears and modeling.

positions and torques in the differential gear box can be described as:

$$
\begin{gathered}
\theta_{l}=L_{p} \theta_{q} \\
\tau_{q}=L_{p}^{T} \tau_{l}
\end{gathered}
$$

with transformation matrix

$$
L_{p}=\left[\begin{array}{cc}
1 & -1 \\
1 & 1
\end{array}\right],
$$

where the link side positions of coupled proximal joint are denoted by vector $\theta_{l}$, while $\theta_{q}$ is joint position vector expressed in joint coordinates. The differential gear box introduces elasticity in the proximal joint. Together with the distal joint position, the transformation matrix for each entire finger can be written as:

$$
\begin{aligned}
\theta_{l} & =L \theta_{q} \\
\tau & =L^{T} \tau_{l}
\end{aligned}
$$

with

$$
\boldsymbol{L}=\left[\begin{array}{ccc}
1 & 0 & 0 \\
0 & 1 & -1 \\
0 & 1 & 1
\end{array}\right] \in \mathbb{R}^{3 \times 3}
$$

where the lower right corner of the transformation matrix is $L_{p} . \theta_{q}$ is represented by $q \in \mathbb{R}^{3}$ from here onward to indicate the position vector of joints after link elasticity and joint coupling. $\tau_{l}$ and $\tau_{q}$ are joint torque vector expressed in joint and motor coordinates, respectively. $\tau_{q}$ is denoted by $\tau$ later in this paper. 


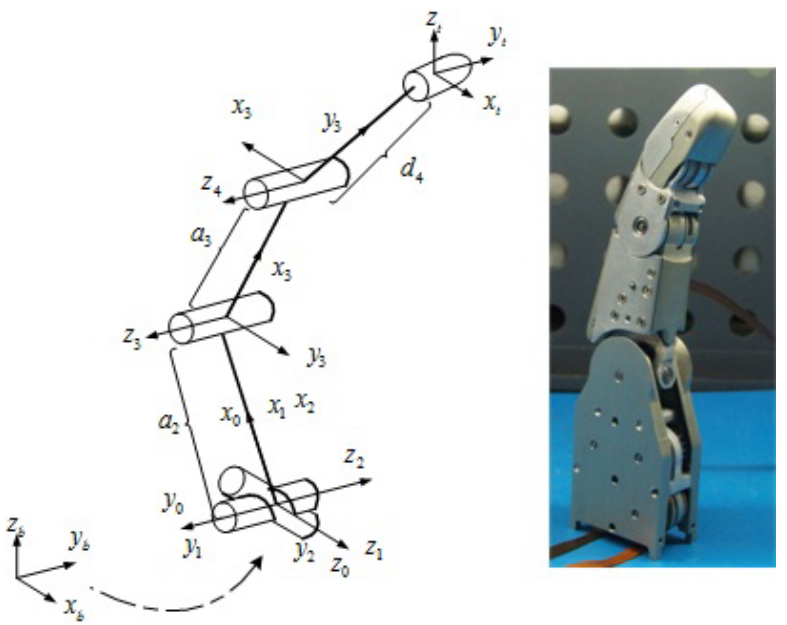

Fig. 4. Frame assignments and parameters in robot finger.

The kinematics model of robot hand with elastic joint and coupling joints then can be expressed as below:

$$
\begin{aligned}
& \boldsymbol{M}(\boldsymbol{q}) \ddot{q}+C(q, \dot{q}) \dot{q}+g(q)=\tau+\tau_{\text {ext }} \\
& \boldsymbol{\Gamma}_{\boldsymbol{m}} \ddot{\theta}_{m}+L^{-T} \tau+\tau_{f, m}=\tau_{m} \\
& \boldsymbol{\tau}=K\left(L q-\theta_{m}\right),
\end{aligned}
$$

where $\boldsymbol{M ( q )}, C(q)$, and $g(q)$ represent the inertia matrices, centrifugal term, and gravity term, respectively. The joint torque vector is given by $K\left(L q-\theta_{m}\right)$, where $\theta_{m}$ indicates the vector of the motor angle divided by the gear ratio $1 / n, L$ denotes transformation matrix for coupling joints as shown in Eq. (8), and $q$ represents the link side joint angle. $K$ and $\Gamma_{m}$ are diagonal matrices that contain the joint stiffness and the motor inertia, respectively, multiplied by the gear ratio squared. $\tau_{\text {ext }}$ and $\tau_{f, m}$ are external torque vector and friction torque vector, respectively. The generalized actuator torque vector, $\tau_{m}$, is considered as the control input. The link frame assignments and parameters are shown in Fig. 4.

\section{Impedance Control Methods for Joint Torque-Based Robot Hand}

\subsection{Joint level impedance control}

The goal of the impedance controller is to achieve a desired dynamic behavior with respect to external forces and torques acting on the link side. As shown in Fig. 2, the dynamic behavior is given by a stiffness parameter $K_{d}$ as well as a damping parameter $D_{d}$. For a robot with elastic joints, this behavior can be realized by a simple PD controller with proportional and derivative gains set as $K_{c}$ and $D_{c}$, 
respectively. The resulting dynamics is influenced by the joint elasticity and the motor inertia. With a negative feedback of the joint torque $\tau$, the apparent inertia (of the rotor) can be scaled down, which means that the closed-loop system reacts to external forces and torques as if the rotor inertia were smaller. ${ }^{18}$ Equation (10) can be rewritten in joint coordinates:

$$
\Gamma \ddot{\theta}+\tau=\tau_{j}
$$

with

$$
\begin{aligned}
\tau_{j} & =L^{T}\left(\tau_{m}-\tau_{f, m}\right) \\
\theta & =L^{-1} \theta_{m} \\
\Gamma & =L^{T} \Gamma_{m} L .
\end{aligned}
$$

Consider a new control variable of the form:

$$
u=\tau+\Gamma_{c} \ddot{\theta},
$$

where $u \in \mathbb{R}^{n}$ is the new input variable and $\Gamma_{c}$ is a diagonal, positive definite matrix. When $\Gamma_{c}<\Gamma, I-\Gamma \Gamma_{c}^{-1}$ becomes negative, thus creating a negative torque feedback. This decreases the virtual motor inertia:

$$
\tau_{j}=\Gamma \Gamma_{c}^{-1} u+\left(I-\Gamma \Gamma_{c}^{-1}\right) \tau .
$$

The noise level of the torque sensors is the main factor in determining the ratio of the scale down $\Gamma_{m} \Gamma_{c}^{-1}$ for the DLR-HIT II hand, where a lower ratio corresponds to a higher stiffness. For passivity consideration, in case that the desired impedance behavior is defined (w.r.t. joint coordinates), a motor position-based PD-controller as following can be used:

$$
u=-K_{c}\left(\theta-\theta_{s}\right)-D_{c} \dot{\theta}
$$

with $K_{c}^{-1}=K_{d}^{-1}-K^{-1}$ and $D_{c}=D_{d}$. $\theta_{s}$ represents the desired configuration. Together with Eqs. (10) and (11), the following closed-loop equations can be formed:

$$
\begin{aligned}
M(q) \ddot{q}+C(q, \dot{q})+g(q) & =\tau+\tau_{\text {ext }} \\
\Gamma_{c} \ddot{\theta}+D_{c} \dot{\theta}+K_{c} \tilde{\theta}+\tau & =0,
\end{aligned}
$$

where $\tilde{\theta}=\theta-\theta_{s}$. The friction is addressed as a part of the external torque for robotic arms. ${ }^{17}$ For robotic hands with compact design and joint coupling, the friction will be addressed explicitly with adaptive friction observer in the following section.

\subsection{Cartesian level impedance control}

As described in the previous section, robot hand dynamics can be expressed in the joint coordinate system by using the transformation matrix $L$. In the following, it is 
assumed that the position and orientation of the end-effector can be described by a set of local coordinates $x \in \mathbb{R}^{m}$, and the relationship between Cartesian coordinates $x$ and the configuration coordinates $q \in \mathcal{Q}$ is given by a known function $f: \mathcal{Q} \rightarrow$ $\mathbb{R}^{m}$, i.e. $x=f(q)$. With the Jacobian $J(q)=\partial f(q) / \partial q$, Cartesian velocities and accelerations can be written as ${ }^{18}$ :

$$
\begin{array}{r}
\dot{x}=J(q) \dot{q} \\
\ddot{x}=J(q) \ddot{q}+\dot{J}(q) \dot{q} .
\end{array}
$$

Throughout this paper, only the nonsingular case is considered, thus it is assumed that the manipulator's Jacobian $J(q)$ has full row rank in the considered region of the workspace.

To specify the desired impedance behavior, the position error $\tilde{x}=x-x_{d}$, between real position $x$ and a virtual equilibrium position (possibly time-varying) $x_{d}$, is introduced. The goal of the impedance controller design here is to alter the system dynamics (9), such that in the presence of external forces and torques at the endeffector $F_{\text {ext }} \in \mathbb{R}^{m}$, a dynamic relationship between $\tilde{x}$ and $F_{\text {ext }}$ could be achieved as follows:

$$
\Lambda_{d} \ddot{\tilde{x}}+D_{d} \dot{\tilde{x}}+K_{d} \tilde{x}=F_{e x t},
$$

where $\Lambda_{d}, D_{d}$, and $K_{d}$ are the symmetric and positive definite matrices of the desired inertia, damping, and stiffness, respectively.

The relationship between the external torque vector $\tau_{\text {ext }}$ and the generalized external force vector $F_{\text {ext }}$ on the end-effector is given by:

$$
\tau_{\text {ext }}=J(q)^{T} F_{\text {ext }} .
$$

Substituting $\ddot{q}=J(q)^{-1}(\ddot{x}-\dot{J}(q)) \dot{q}$ from Eqs. (19) and Eq. (21) into (9) leads to

$$
M(q) J(q)^{-1}(\ddot{x}-\dot{J}(q) \dot{q})+C(q, \dot{q}) \dot{q}+g(q)=\tau+J(q)^{T} F_{\text {ext }} .
$$

With $\dot{q}=J(q)^{-1} \dot{x}$ from Eq. (18), and multiplying the resulting equation by $J(q)^{-T}$, the relationship between the Cartesian coordinates $x$ and the joint torques $\tau$ can now be expressed in the following form:

$$
\Lambda(x) \ddot{x}+\mu(x, \dot{x}) \dot{x}+J(q)^{-T} g(q)=J(q)^{-T} \tau+F_{\text {ext }},
$$

where the matrices $\Lambda(x)$ and $\mu(x, \dot{x})$ are given by:

$$
\begin{aligned}
\Lambda(x) & =J(q)^{-T} M(q) J(q)^{-1} \\
\mu(x, \dot{x}) & =J(q)^{-T}\left(C(q, \dot{q})-M(q) J(q)^{-1} \dot{J}(q)\right) J(q)^{-1}
\end{aligned}
$$

with $q=f^{-1}(x)$ and $\dot{q}=J\left(f^{-1}(x)\right) \dot{x}$.

By treating them as the external torques, the gravity torque $g(q)$ and the joint torque $\tau$ can be rewritten in the form of the equivalent task space gravity 
forces $F_{g}(x)$ and the new input vector $F_{\tau}$. Therefore, the system equations can be expressed in the following form:

$$
\Lambda(x) \ddot{x}+\mu(x, \dot{x}) \dot{x}+F_{g}(x)=F_{\tau}+F_{\text {ext }} .
$$

$\Lambda(x)$ and $\mu(x, \dot{x})$ are the inertia matrix and the Coriolis/centrifugal matrix with respect to the coordinates $x$, respectively. Combining Eqs. (26) and (20), the impedance control law, which is the desired closed-loop system, with $F_{\tau}$ as the control input, can be arrived:

$$
\begin{aligned}
F_{\tau}= & \Lambda(x) \ddot{x}_{d}+\mu(x, \dot{x}) \dot{x}+\left(\Lambda(x) \Lambda_{d}^{-1}-I\right) F_{e x t}+F_{g}(x) \\
& -\Lambda(x) \Lambda_{d}^{-1}\left(D_{d} \dot{\tilde{x}}+K_{d} \tilde{x}\right) .
\end{aligned}
$$

By selecting the desired torque vector $\tau$ as:

$$
\tau=J(q)^{T} F_{\tau}+C(q, \dot{q}) \dot{q}-J(q)^{T} \Lambda(x) \dot{J}(q) J(q)^{-1},
$$

Eq. (27) can be simplified as:

$$
\begin{aligned}
F_{\tau}= & \Lambda(x) \ddot{x}_{d}-\Lambda(x) \Lambda_{d}^{-1}\left(D_{d} \dot{\tilde{x}}+K_{d} \tilde{x}\right)+F_{g}(x) \\
& +\left(\Lambda(x) \Lambda_{d}^{-1}-I\right) F_{\text {ext }}
\end{aligned}
$$

with the assumption that centripetal and Coriolis forces can be ignored at the robot's relatively low operating speeds. Furthermore, if the desired inertia $\Lambda_{d}$ is chosen as identical to the robot inertia $\Lambda(x)$, the feedback of external forces $F_{\text {ext }}$ can be avoided. Then, it follows that the actual implementation of the impedance controller:

$$
F_{\tau}=\Lambda(x) \ddot{x}_{d}-D_{d} \dot{\tilde{x}}-K_{d} \tilde{x}+F_{g}(x) .
$$

As a result, the desired joint torques $\tau$ can be expressed as:

$$
\tau=g(q)+J(q)^{T}\left(\Lambda(x) \ddot{x}_{d}-D_{d} \dot{\tilde{x}}-K_{d} \tilde{x}\right) .
$$

Using motor $\theta$ instead of the link side angles $q$ in the forward kinematics $x=f(q)$, impedance controller based on PD position control w.r.t. Cartesian coordinates can be generalized from Eq. (15). Then, the feedback law is given by:

$$
\begin{aligned}
u & =-J(\theta)^{T}\left(K_{x} \tilde{x}(\theta)+D_{x} \dot{x}\right) \\
\tilde{x}(\theta) & =f(\theta)-x_{d} \\
\dot{x} & =J(\theta) \dot{\theta}
\end{aligned}
$$

with $K_{x}$ and $D_{x}$ representing the desired stiffness and damping matrices, respectively, corresponding to $K_{d}$ and $D_{d}$ in Eq. (32). $x_{d}$ indicates the virtual motor side position in Cartesian coordinates. The controller in Eq. (33), together with Eq. (14), 
forms the closed-loop system:

$$
\begin{aligned}
M(q) \ddot{q}+C(q, \dot{q})+g(q) & =\tau+\tau_{\text {ext }} \\
\Gamma_{c} \ddot{\theta}+J(\theta)^{T}\left(K_{x} \tilde{x}(\theta)+D_{x} \dot{x}\right)+\tau & =0 .
\end{aligned}
$$

\section{Friction Observer with Extended Kalman Filter and Gravity Compensation}

\subsection{Friction model linearization and parameters estimation with Least Squares}

The performance of the controller can be significantly improved by obtaining the appropriate robot dynamic model. Due to the compact design and joint coupling, the friction model on the finger joints is difficult to identify. Furthermore, joint friction parameters could vary from finger to finger due to components tolerance. Therefore, a simple friction model with viscous and static friction is chosen in this paper, which is described as:

$$
\tau_{f, m}=b \dot{\theta}+c \cdot \operatorname{sign}(\dot{\theta}),
$$

where $b$ and $c$ are viscous friction coefficient and static friction coefficient, respectively. The dynamic model of a single robot joint is expressed from Eq. (10) as:

$$
I_{m} \ddot{\theta}=\tau_{m c}-b \dot{\theta}-c \cdot \operatorname{sign}(\dot{\theta}),
$$

where $\tau_{m c}=\tau_{m}-L^{-T} \tau$ denotes the sum of torques acting on the motor except friction. We use $\tau$ instead of $\tau_{m c}$ through this whole section for the sake of simplicity. $I_{m}$ represents the motor inertia. In order to implement controller with friction compensation, it is necessary to determine parameters corresponding to the robot dynamical model. Nonlinearity of the above friction model resides in the signum function term $\operatorname{sign}(\dot{\theta})$. By multiplying both sides of the Eq. (36) with term $\dot{\theta}$, the friction model is given by linear form:

$$
I_{m} \dot{\theta} \ddot{\theta}+b \dot{\theta}^{2}+c \underbrace{\dot{\theta} \operatorname{sign}(\dot{\theta})}_{|\dot{\theta}|}=\dot{\theta} \tau .
$$

By integrating Eq. (37) over one timestep $t_{s}=t_{k+1}-t_{k}$ with a trapzoidal approximation, the Least Squares estimation can be achieved:

$$
\underbrace{\left[\begin{array}{cc}
\frac{1}{2}\left(\dot{\theta}_{k+1}^{2}-\dot{\theta}_{k}^{2}\right) & \frac{t_{s}}{2}\left(\dot{\theta}_{k+1}^{2}+\dot{\theta}_{k}^{2}\right) \\
\downarrow k=0 \ldots N & \frac{t_{s}}{2}\left(\left|\dot{\theta}_{k+1}^{2}\right|+\left|\dot{\theta}_{k}^{2}\right|\right) \\
\vdots
\end{array}\right]}\left[\begin{array}{c}
I_{m} \\
b \\
c
\end{array}\right]=\left[\begin{array}{c}
\frac{t_{s}}{2}\left(\tau_{k+1} \dot{\theta}_{k+1}-\tau_{k} \dot{\theta}_{k}\right) \\
\downarrow k=0 \ldots N \\
\vdots
\end{array}\right] .
$$




\subsection{Friction estimation with extended Kalman filter}

Kalman filter is a powerful estimation tool when the precise nature of the modeled system is unknown, which can be used to fulfill the nonlinear system parameter estimation for DLR-HIT dexterous hand II. The proposed controller design with extended Kalman filter is described in this section. The extended Kalman filter is subdivided into prediction step and estimation step. ${ }^{20}$ Based on the current state and the dynamic model of the system, a forecast can be calculated for the state in the prediction step:

$$
\begin{aligned}
\Delta \hat{x}_{k+1}^{-} & =A_{k} \Delta \hat{x}_{k}^{+}+B_{K} u_{k} \\
P_{k+1}^{-} & =A_{k} P_{k}^{+}+G_{K} Q_{k} G_{k}^{T} .
\end{aligned}
$$

The estimation step is defined, where the forecast and the measurements are compared, and an optimal compromise is made:

$$
\begin{aligned}
K_{k} & =P_{k}^{-} H_{k}^{T}\left(H_{k} P_{k}^{-} H_{k}^{T}+R_{k}\right)^{-1} \\
\Delta \hat{x}_{k}^{+} & =\Delta \hat{x}_{k}^{-}-K\left(H_{k} \Delta \hat{x}_{k}^{-}-\Delta y_{k}\right) \\
P_{k}^{+} & =\left(I-K_{k} H_{k}\right) P_{k}^{-} .
\end{aligned}
$$

In order to eliminate a possible divergence of $\Delta x$, the error state is set accordingly to zero after the estimation step.

As shown in Connette's work, ${ }^{21}$ to derive an adaptive extended Kalman filter, viscous and static friction parameters $b$ and $c$ are modeled as constant system states. $\tau$ is considered as a state variable, or specifically a measurement variable rather than an input variable. The influence of $\tau$ can be adjusted by tuning its corresponding system noise parameter. Replacing $I_{m}$ with $I$ for simplicity, the dynamic model of the system can be expressed as:

$$
\frac{d}{d t} \underbrace{\left(\begin{array}{c}
\theta \\
\dot{\theta} \\
\tau \\
b \\
c
\end{array}\right)}_{:=x}=\underbrace{\left(\begin{array}{c}
\dot{\theta} \\
\frac{1}{I_{m}}(\tau-b \dot{\theta}-\operatorname{csign}(\dot{\theta})) \\
0 \\
0 \\
0
\end{array}\right)}_{:=a} .
$$

The parameters describing viscous and static frictions are estimated along with position, velocity, and $\tau$. Therefore, further nonlinearities besides the items already mentioned are introduced into the system, by partially deriving the system dynamics equation, replacing the partial derivation sign $\partial$ with differences sign $\Delta$ and using linearlized form of sign function:

$$
f(\dot{\theta})=\left\{\begin{array}{cc}
-1 & \dot{\theta}<- \text { limit } \\
\frac{1}{\text { limit }} & - \text { limit }<\dot{\theta}<\text { limit } \\
1 & \text { limit }<\dot{\theta}
\end{array}\right.
$$


where nonlinearities in the system dynamic model can be eliminated. The following linearized system dynamics equation can be arrived:

$$
\frac{d}{d t}\left(\begin{array}{c}
\Delta \theta \\
\Delta \dot{\theta} \\
\Delta \tau \\
\Delta b \\
\Delta c
\end{array}\right)=A_{l i n} \cdot\left(\begin{array}{c}
\Delta \theta \\
\Delta \dot{\theta} \\
\Delta \tau \\
\Delta b \\
\Delta c
\end{array}\right)
$$

where:

$$
A_{l i n}=\left(\begin{array}{ccccc}
0 & 1 & 0 & 0 & 0 \\
0 & -\left(\frac{b}{I_{m}}+\alpha \frac{c}{I_{m}}\right) & \frac{1}{I_{m}} & \frac{-\dot{\theta}}{I_{m}} & \frac{-f(\dot{\theta})}{I_{m}} \\
0 & 0 & 1 & 0 & 0 \\
0 & 0 & 0 & 1 & 0 \\
0 & 0 & 0 & 0 & 1
\end{array}\right)
$$

with $\alpha$ represents the derivation of $f(\dot{\theta})$, given by:

$$
\alpha=\left\{\begin{array}{cc}
0 & \dot{\theta}<- \text { limit } \\
\frac{1}{\text { limit }} & - \text { limit }<\dot{\theta}<\text { limit } . \\
0 & \text { limit }<\dot{\theta}
\end{array}\right.
$$

The solution to the differential equations (41) can be expressed ${ }^{22}$ as:

$$
\begin{aligned}
X(t) & =\Phi\left(t, t_{0}\right) \cdot X\left(t_{0}\right) \\
\Phi\left(t, t_{0}\right) & =e^{A_{l i n}\left(t-t_{0}\right)} .
\end{aligned}
$$

The discrete-time-space solution is achieved by calculating Eq. (43) from discrete time $k \cdot t_{s}$ to $(k+1) \cdot t_{s}$ and expanding the exponential function in its power series:

$$
\begin{aligned}
X_{k+1} & =A_{k} \cdot X_{k} \\
A_{k} & =1+A_{l i n, k} \cdot \frac{t_{s}}{1 !}+A_{l i n, k}^{2} \cdot \frac{t_{s}}{2 !}+\cdots,
\end{aligned}
$$

where $A_{l i n, k}=A_{l i n}\left(x\left(k t_{s}\right)\right)=A_{l i n}$. Together with Eq. (42), the adaptive estimation with linearized extended Kalman filter results in:

$$
\Delta x_{k+1}=\left(\begin{array}{ccccc}
1 & a_{2} & a_{3} & -\dot{\theta} \cdot a_{3} & -f(\dot{\theta}) \cdot a_{3} \\
0 & a_{1} & \frac{1}{I_{m}} \cdot a_{2} & -\frac{\dot{\theta}}{I_{m}} \cdot a_{2} & -\frac{f(\dot{\theta})}{I_{m}} \cdot a_{2} \\
0 & 0 & 1 & 0 & 0 \\
0 & 0 & 0 & 1 & 0 \\
0 & 0 & 0 & 0 & 1
\end{array}\right) \cdot \Delta x_{k},
$$


where:

$$
\begin{aligned}
& a_{1}=e^{-(b+\alpha c) \cdot \frac{t_{s}}{I_{m}}} \\
& a_{2}=\frac{I_{m}}{b+\alpha c} \cdot\left(1-e^{-(b+\alpha c) \cdot \frac{t_{s}}{I_{m}}}\right) \\
& a_{3}=\frac{I_{m}}{(b+\alpha c)^{2}} \cdot\left(e^{-(b+\alpha c) \cdot \frac{t_{s}}{I_{m}}}-1+(b+\alpha c) \frac{t_{s}}{I_{m}}\right) .
\end{aligned}
$$

Considering that the system parameters $\mathrm{b}$ and $\mathrm{c}$ are constant and estimated with Least Squares in the prior section, the nonadaptive estimation with extended Kalman filter can also be achieved by solving Eq. (41):

$$
\Delta x_{k+1}=\left(\begin{array}{ccc}
1 & a_{2} & a_{3} \\
0 & a_{1} & \frac{1}{I_{m}} \cdot a_{2} \\
0 & 0 & 1
\end{array}\right) \cdot \Delta x_{k} .
$$

Figure 5 illustrates the experimental results of viscous and static friction parameters estimated with extended Kalman filter. The estimations converge toward the assumed values $(b=0.3, c=0.2)$ calculated with the Least Squares technique as shown in the figure, which indicates the effectiveness of the proposed friction estimation method with extended Kalman filter. The consistent errors, especially with the estimation of $c$, can be explained with the necessary approximations and linearizations during the filter-design. The system noise $Q$ and the measurement noise covariance $R$ play a crucial role in the performance of the Kalman filter. Superior filter performance can be obtained by tuning the filter parameters $Q$ and $R$ offline. The behavior of the friction observer can also be observed in the velocity estimation. Due to the compact design and complex transmission system, the friction parameters are hardly identified with known friction models and could be varying depending on different joints and fingers. Therefore, the velocity estimation with adaptively estimated system parameters shows better results than the velocity

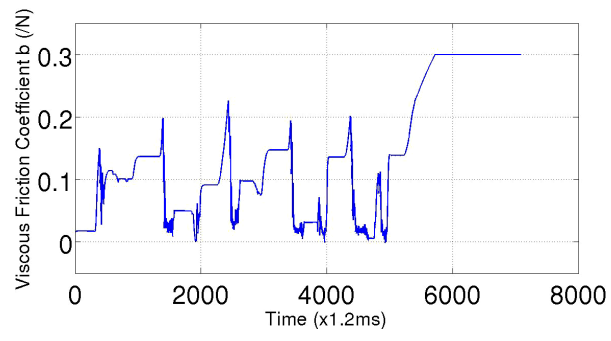

(a)

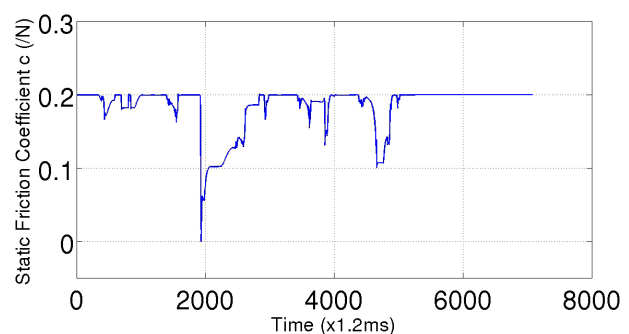

(b)

Fig. 5. Friction parameter estimation with EKF: (a) viscous friction parameter b and (b) static friction parameter $\mathrm{c}$. 


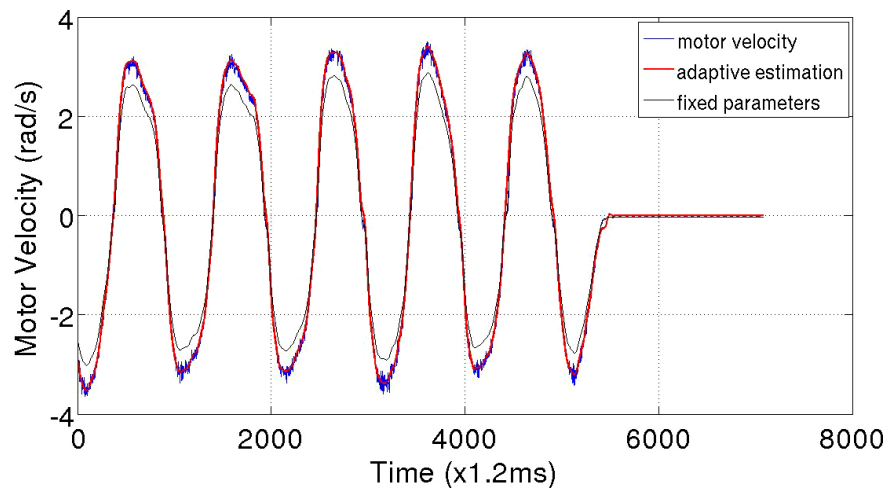

Fig. 6. Velocity estimation with EKF.

estimation with preidentified system parameters based on Least Squares, as shown in Fig. 6.

\subsection{A practical gravity compensation methods}

As shown in Tomei's work, ${ }^{23}$ the gravity torque compensation term in the desired steady state can be used for a motor position-based PD-controller. The gravity forces on the robot hand can be expressed as:

$$
\begin{aligned}
G(\theta) & =\frac{\partial V(\theta)}{\partial \theta} \\
V(\theta) & =\sum_{i=1}^{n} m_{i} \times g h_{i}(\theta),
\end{aligned}
$$

where $V: \mathbb{R}^{n} \rightarrow \mathbb{R}$ is the potential energy of the robot hand. $\theta$ denotes the joint angle vector. $h_{i}$ represents the height of the mass center, which can be obtained by using the forward kinematics map.

Due to the integrated design of the DLR-HIT II dexterous robot hand, the gravity force for each joint cannot be easily calculated explicitly with the nominal mass and inertiae of the robot. Instead the estimated gravity compensation is implemented with forward kinematics of the robot hand in this paper. In order to obtain the gravity forces estimation, Eq. (47) can be manipulated as:

$$
G(\theta)=H(\theta) \cdot M \times g,
$$

where $H(\theta), g$ represent mass center coordinates vector and gravity vector with respect to base frame, as shown in Sec. 4. $M$ indicates the mass vector that can be estimated by solving the following Least Squares equation:

$$
\min _{M} \frac{1}{2}\|H(\theta) \cdot M-G(\theta)\|_{2},
$$


where $G(\theta)$ can be obtained from the torque sensors explicitly with the finger in different configurations. However, large deviations from the steady-state positions may occur in case of low desired stiffness with elastic joints. By using iteration methods, ${ }^{24}$ gravity compensation based on motor position can be implemented to compensate for link side gravity.

\section{Experimental Results}

\subsection{Friction estimation}

Since friction compensation is achieved in joint space, the experiments of the proposed friction observer are conducted with a PD-based joint impedance controller as Eq. (15). Relevant parameters of the robot and coefficients needed in friction compensation are estimated as described in the prior section and listed in Table 1.

Table 1. Control parameters.

\begin{tabular}{lcccc}
\hline Parameters & $\begin{array}{c}\text { Length } \\
(\mathrm{mm})\end{array}$ & $\begin{array}{c}\text { Estimated mass } \\
(\mathrm{kg})\end{array}$ & $\begin{array}{c}\text { Mass center } \\
(\mathrm{mm})\end{array}$ & $\begin{array}{c}\text { Estimated inertia } \\
\left(\mathrm{kg} \cdot \mathrm{mm}^{2}\right)\end{array}$ \\
\hline Joint 1 & 0 & 0.0232 & 27.49 & 17.53 \\
Joint 2 & 55 & 0.0232 & 27.49 & 17.53 \\
Joint 3 & 25 & 0.0158 & 2.5 & 0.098 \\
Joint 4 & 15 & 0.0137 & 3 & 0.1233 \\
\hline
\end{tabular}
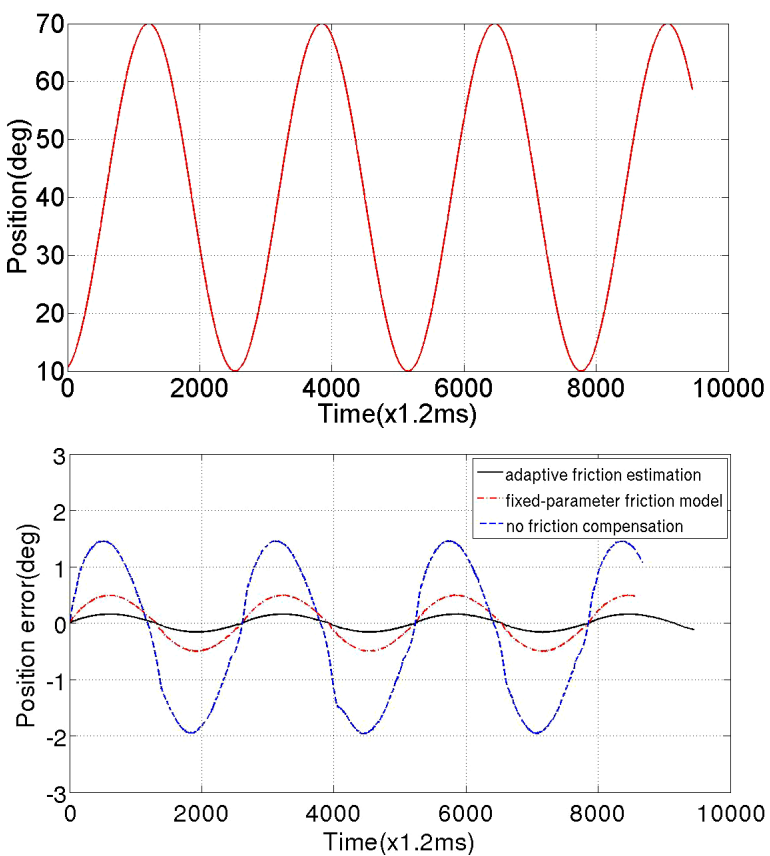

Fig. 7. Position tracking experiments with and without friction and gravity compensation. 
The third joint of the robot finger follows a periodic trajectory in order to demonstrate the position-tracking ability of the proposed impedance controller with friction observer on the modular DLR-HIT II dexterous robot finger. As shown in Fig. 7 , the position error is less than $0.16^{\circ}$ with adaptive friction and optimal gravity compensation, comparing favorably to $1.5^{\circ}$ without friction compensation, and $0.5^{\circ}$ for fixed-parameters friction and gravity compensation. Thus, it can be concluded that improved performance is achieved by the proposed impedance controller with friction observer.

\subsection{Joint impedance control}

In this section, the experiments of the proposed impedance controller are conducted in joint space. Each joint of the finger is independently driven in the experiments. The aim is to move all three joints of the finger in different directions, coming in contact with an external object before reaching the desired position. Damping and stiffness are set at $D_{d}=[0.0013,0.0013,0.0031]\left(N \cdot m \cdot s /{ }^{\circ}\right)$, $K_{d}=[0.0625,0.0625,0.125]\left(N \cdot m /{ }^{\circ}\right)$. Figure 8 shows all the finger joints tracking
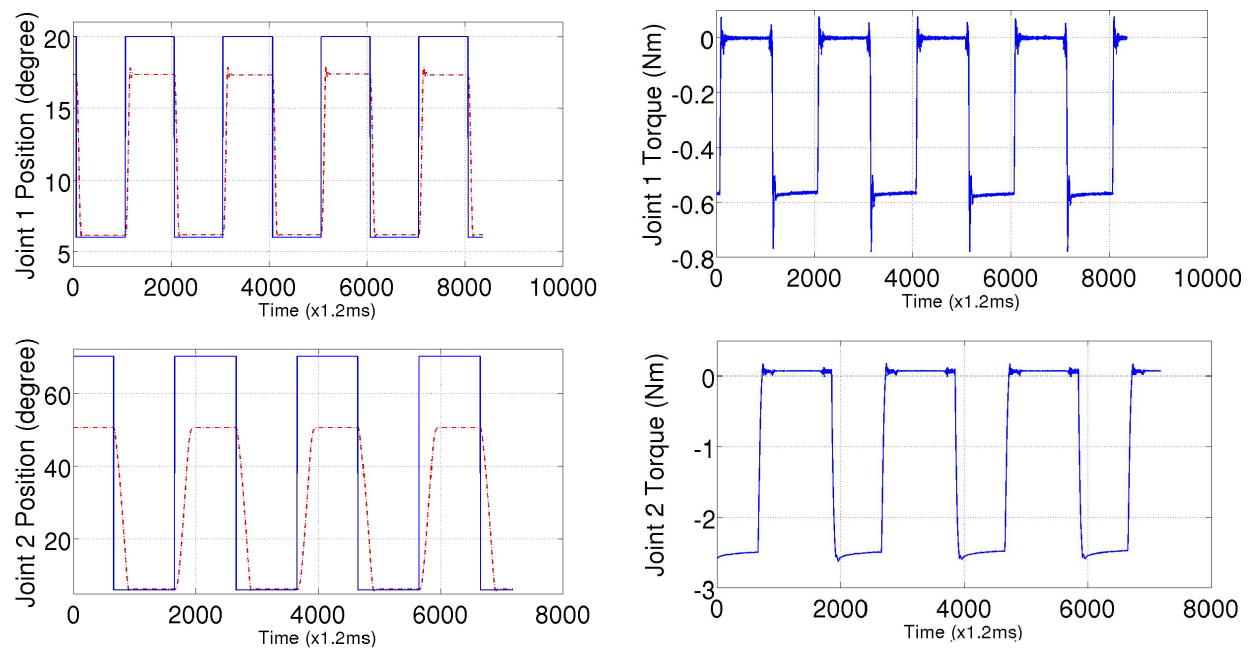

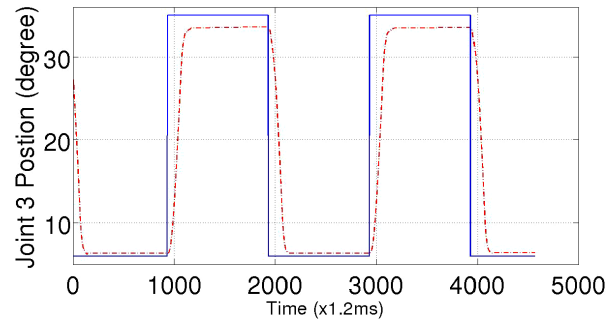

(a)

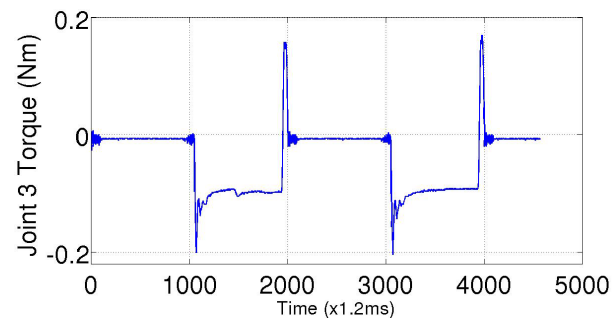

(b)

Fig. 8. Position tracking and force response in abduction (joint 1), proximal (joint 2), and distal (joint3) joints of the finger: (a) position tracking and (b) torque response. 


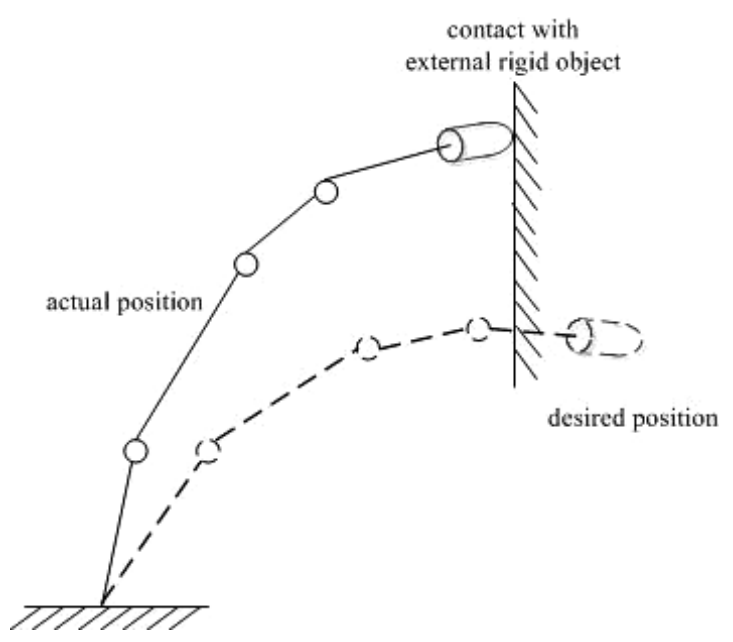

Fig. 9. Robot finger contacts with external rigid object.

the desired position trajectory (red dash line). As shown in Fig. 9, contact is made with a rigid external object at joint angles of $\left[28^{\circ}, 52^{\circ}, 17.5^{\circ}\right]$ (distal, proximal, and abduct joints, respectively), where real tracking separates from the desired tracking. The experimental results demonstrate that the joints can follow the desired trajectory closely in the free space, and the joint torques increase stably as the fingers make contact with the environment. It is, therefore, concluded that the finger joint space impedance control behavior is successfully achieved.

\subsection{Cartesian impedance control}

For the Cartesian impedance control law (30)-(34), the following control parameters $\Lambda(x), g(q), K_{x}$, and $D_{x}$ should first be obtained. As described in Sec. $4, g(q)$ as well as $\Lambda(x)$ can be estimated by using Least Squares techniques and iteration methods based on motor side inertia instead of link side inertia. $D_{x}=12.5(\mathrm{~N} \cdot \mathrm{s} / \mathrm{m})$ and $K_{x}=900(\mathrm{~N} / \mathrm{m})$ are designed by the double-diagonalization approach with the robot inertia matrix and the desired damping ratio. ${ }^{25}$

The Cartesian impedance control experiment has been made on a single finger of the robot hand while the other fingers are broken. As shown in Fig. 10, the joint tracks the desired position trajectory (red line), with real tracking curve shown as the solid line. Contact is made with a rigid environment where the position offset $\Delta x=0.0115 \mathrm{~m}$ in the $x$ direction. The filtered joint torque can also be found in Fig. 10. The experiment results show that the joint can follow the desired trajectory closely in the free space and the joint torque increases stably while it makes contact with the environment.

In a further experiment, the robot pauses at a virtual equilibrium position $x_{d}=[0.036,0.036,0.2] \mathrm{m}$ w.r.t. Cartesian coordinates. In the first part of this experiment, the endpoint of the finger is pulled and then released, as shown in Fig. 11. 

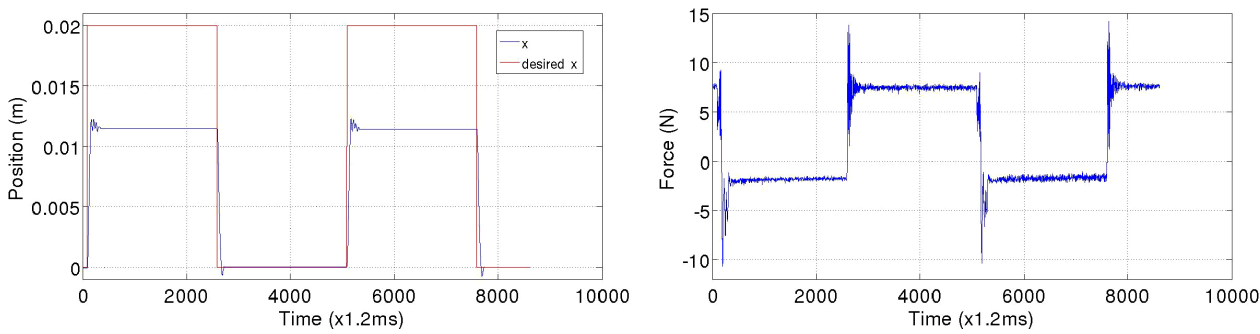

Fig. 10. Cartesian position and force tracking with contacting environment.
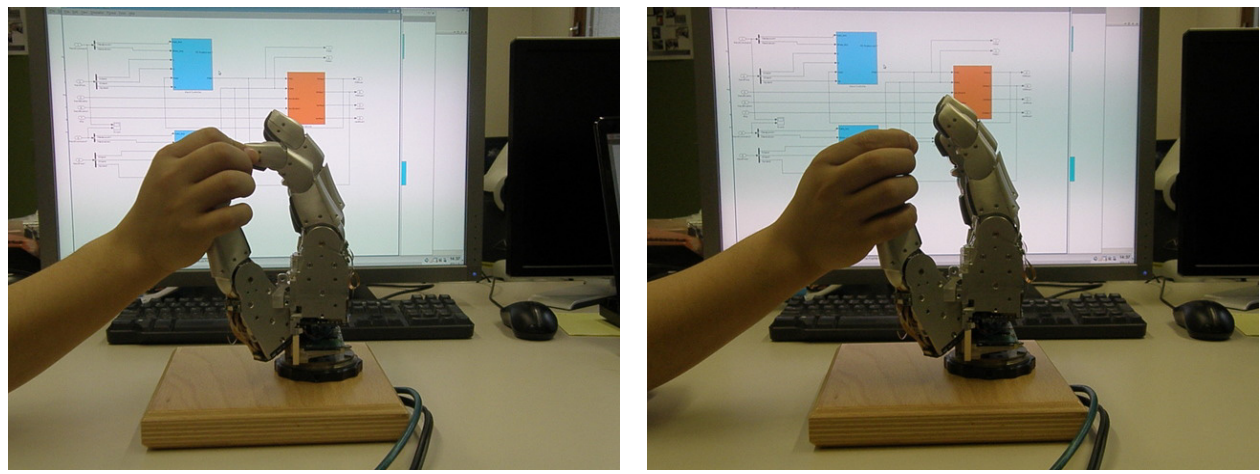

Fig. 11. Pull the endpoint of the robot finger and release it.
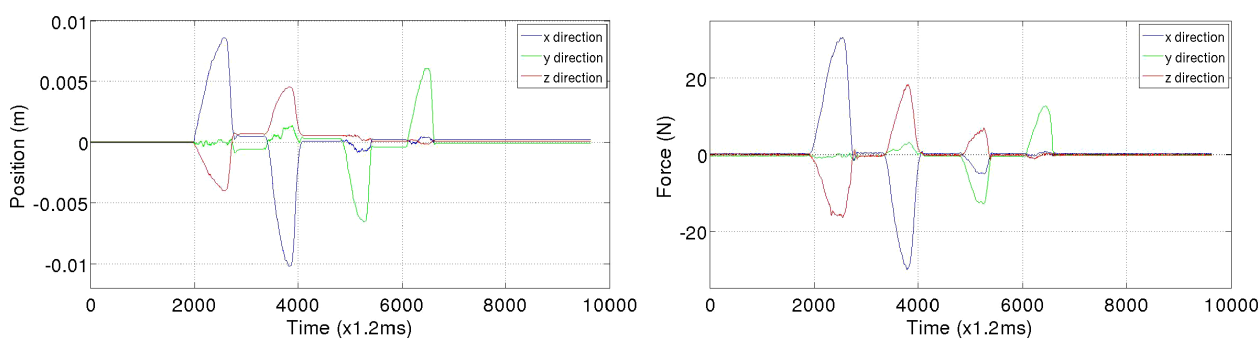

Fig. 12. Robot finger Cartesian coordinate position and force response to external forces.

The fingertip Cartesian position displacement and force response of the proposed Cartesian impedance controller are recorded, and illustrated as Fig. 12. The robot overcomes the gravity and friction, returning to the $x_{d}$ as soon as the external force is released. With the friction and gravity compensation proposed in this paper, the static error in the $x$ axis is reduced to less than $0.2 \mathrm{~mm}$, as well as $y$ and $z$ directions. In the second part of the experiment, back and forth forces in the $x$ direction are applied to the fingertip. Figure 13 shows the measurements of stiffness comparing with the desired stiffness $\left(K_{x}=1,300(\mathrm{~N} / \mathrm{m}), D_{x}=12.5(\mathrm{~N} \cdot \mathrm{s} / \mathrm{m})\right)$. The hysteresislike deviation from the desired stiffness slope is due to Cartesian damping term. 

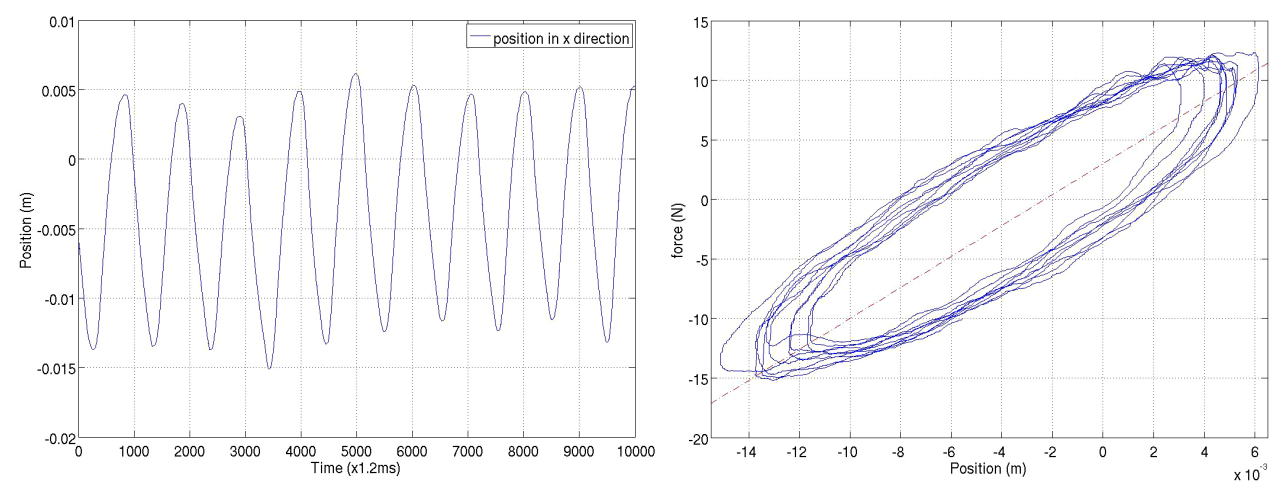

Fig. 13. Position versus force in $x$-direction. The linear slope (dash dot line) is the desired stiffness.

It can, therefore, be concluded that the Cartesian impedance behavior is successfully achieved.

\section{Conclusions}

In this paper, joint and Cartesian impedance controllers with adaptive friction observer and optimal gravity compensation for dexterous robot hand DLR-HIT II with elastic joint are proposed. Robot hand modeling is presented for further controller investigation. Impedance control based on joint torque feedback is implemented and evaluated in this paper. In order to address friction due to the compact design, complex transmission system and joint coupling, an adaptive friction observer is proposed with extended Kalman filter based on a basic friction model. The performance of the adaptive friction estimation method is analyzed and compared to the friction estimation with preidentified system parameters. Estimated gravity is used in this work to address uncertainties in gravity compensation, which is based on Least Squares techniques and implemented to compensate for the link side gravity. An FPGA hardware and software structure with multisensory system and real-time communication is designed for the practical implementation of the proposed controller. Impedance control experiments are conducted with the five-finger dexterous robot hand DLR-HIT II in joint and Cartesian coordinate systems, successfully demonstrating the effectiveness of the proposed controller and hardware architecture. Experimental results show improved position tracking performance of the impedance controller with the aid of adaptive friction estimation and optimal gravity compensation.

\section{References}

1. J. Salisbury and J. Craig, "Articulated hands: force control and kinematic issues," The International Journal of Robotics Research 1(1) (1982) 4.

2. S. Jacoben et al., "The Utah/MIT dexterous hand: Work in progress," International Journal of Robotics and Research 3(4) (1984) 21-50. 
3. C. Lovchik and M. Difler, "The robonaut hand: A dextrous robotic hand for space," in Proceedings of the IEEE International Conference on Robotics and Automation (1999), pp. 907-912.

4. H. Kawasaki, T. Komatsu and K. Uchiyama, "Dexterous anthropomorphic robot hand with distributed tactile sensor: Gifu hand II, IEEE," ASME Transaction on Mechatronics 7(3) (2002) 296-303.

5. J. Butterfass, M. Grebenstein and H. Liu, "DLR-Hand II: next generation of a dexterous robot hand," In Proceedings of the 2001 IEEE International Conference on Robotics ES Automation (2001), pp. 109-114.

6. H. Liu, P. Meusel, G. Hirzinger, M. Jin, Y. Liu and Z. Xie, "The modular multisensory DLR-HIT-hand: hardware and software architecture," IEEE/ASME Transactions on Mechatronics 13(4) Part 0 (2008) 461-469.

7. N. Hogan, "Impedance control an approach to manipulation. I-Theory. IIImplementation. III-Applications," ASME, Transactions, Journal of Dynamic Systems, Measurement, and Control (ISSN 0022-0434) 107 (1985).

8. R. Bonitz and T. Hsia, "Internal force-based impedance control for cooperating manipulators," IEEE Transactions on Robotics and Automation 12(1) (1996) 78-89.

9. S. Schneider and R. Cannon Jr., "Object impedance control for cooperative manipulation: theory and experimental results," IEEE Transactions on Robotics and Automation 8(3) (1992) 383-394.

10. L. Biagiotti, H. Liu, G. Hirzinger and C. Melchiorri, "Cartesian impedance control for dexterous manipulation," in Proceedings of the IEEE/RSJ International Conference on Intelligent Robots and Systems, pp. 3270-3275.

11. R. Wei, X. Gao, M. Jin, Y. Liu, H. Liu, N. Seitz, R. Gruber and G. Hirzinger, "FPGA based hardware architecture for HIT/DLR hand," in 2005 IEEE/RSJ International Conference on Intelligent Robots and Systems, 2005 (IROS 2005) (2005), pp. 523-528.

12. N. Kircanski and A. Goldenberg, "An experimental study of nonlinear stiffness, hysteresis, and friction effects in robot joints with harmonic drives and torque sensors," The International Journal of Robotics Research 16(2) (1997) 214.

13. H. Liu, Y. Liu, M. Jin, K. Sun and J. Huang, "An experimental study on cartesian impedance control for a joint torque-based manipulator," Advanced Robotics 22(11) (2008) 1155-1180.

14. L. Le Tien, A. Albu-Schaeffer, A. De Luca and G. Hirzinger, "Friction observer and compensation for control of robots with joint torque measurement," 1 (2008) 37893795 .

15. M. Spong, K. Khorasani and P. Kokotovic, "An integral manifold approach to the feedback control of flexible joint robots," Robotics and Automation, IEEE Journal of [Legacy, Pre-1988] 3(4) (1987) 291-300.

16. A. De Luca and P. Lucibello, "A general algorithm for dynamic feedback linearization of robots with elastic joints," in IEEE International Conference on Robotics and Automation (Institute of Electrical Engineers Inc. (IEEE), 1998), pp. 504-510.

17. A. Albu-Schaeffer, C. Ott and G. Hirzinger, "A unified passivity-based control framework for position, torque and impedance control of flexible joint robots," The International Journal of Robotics Research 26(1) (2007) 5-21.

18. C. Ott, A. Albu-Schaeffer, A. Kugi and G. Hirzinger, "On the Passivity-Based Impedance Control of Flexible Joint Robots," IEEE Transactions on Robotics 24(2) (2008) 416-429.

19. M. Good, L. Sweet and K. Strobel, "Dynamic models for control system design of integrated robot and drive systems," Journal of Dynamic Systems, Measurement, and Control 107 (1985) 53.

20. A. Gelb, Applied Optimal Estimation (MIT press, Cambridge, MA, 2002). 
21. C. P. Connette, Intern Report On DLR Hand II (Institute of Robotic and Mechatronics, DLR, 2006).

22. R. Brown and P. Hwang, Introduction to Random Signals and Applied Kalman Filtering (Wiley, New York, 1992).

23. P. Tomei, "A simple PD controller for robots with elastic joints," IEEE Transactions on Automatic Control 36(10) (1991) 1208-1213.

24. C. Ott, A. Albu-Schaeffer, A. Kugi, S. Stramigioli and G. Hirzinger, "A passivity based Cartesian impedance controller for flexible joint robots - Part I: torque feedback and gravity compensation," in IEEE International Conference on Robotics and Automation (Citeseer, 2004), pp. 2659-2665.

25. A. Albu-Schaeffer, C. Ott and G. Hirzinger, "A passivity based Cartesian impedance controller for flexible joint robots - Part II: full state feedback, impedance design and experiments," in IEEE International Conference on Robotics and Automation. (Citeseer, 2004), pp. 2666-2672.

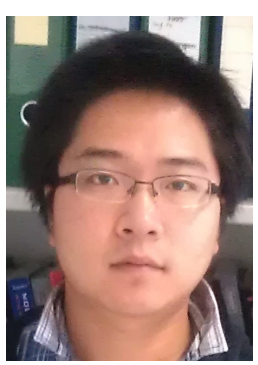

Zhaopeng Chen received his B.S. degree in mechanical engineering and automation and M.S. degree in mechatronic engineering from Harbin Institute of Technology, Harbin, P.R. China, in 2005 and 2007, respectively. From 2008 to 2010, he was a joint-train Ph.D. candidate in the Institute of Robotics and Mechatronics, DLR German Aerospace Center, Oberpfaffenhofen, Germany. Since 2010, he has been with DLR German Aerospace Center in Oberpfaffenhofen, Germany, as a research scientist. His current interests include dexterous robot hands and nonlinear control.

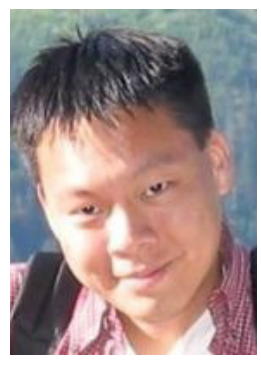

Neal Y. Lii received his B.S. degree in aeronautics and astronautics in 1995 from Purdue University, West Lafayette, IN, USA, M.S. degree in mechanical engineering in 1999 from Stanford University, Palo Alto, CA, USA, and Ph.D. degree in engineering (electrical engineering division) in 2009 from the University of Cambridge, Cambridge, UK.

He has held industrial positions in North America and Europe working the area of mechatronics. He worked on a variety of automotive applications ranging from driver assistance, powertrain control systems, to drive-by-wire systems, the latter of which was carried out at BMW AG in Munich, Germany. He has been with DLR German Aerospace Center in Oberpfaffenhofen, Germany since 2009, where he coordinates and participates in several projects in humanoid robotic hands, space robotics, and autonomous electrical vehicles. 


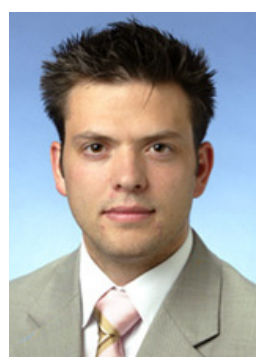

Thomas Wimböck studied electrical engineering at the Technical University of Munich (TUM) with visits to the Rensselaer Polytechnic Institute, Troy, and to the École Polytechnique Fédérale de Lausanne, Switzerland. In 2004, he received his B.Sc. and M.Sc. degrees in electrical engineering from TUM. Subsequently, he joined the DLR, Institute of Robotics and Mechatronics, as a research scientist. His main research interests include nonlinear control, dexterous robot hands, impedance control, VS control, and two-handed (humanoid) manipulation. He received together with his colleagues, several scientific awards: Industrial Robot Outstanding Paper Award 2007, International Conference on Robotics and Automation Best Video Award 2007 and 2009.

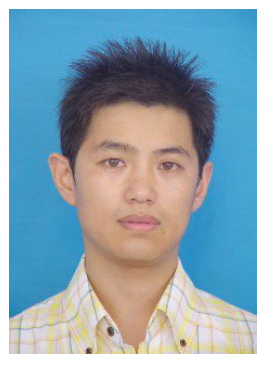

Shaowei Fan received his B.Eng., M.Eng., and Ph.D. degrees in mechatronics engineering from Harbin Institute of Technology, Harbin, China, in 2001, 2005, and 2010, respectively.

Since 2005, he has been with the State Key Laboratory of Robotics and System, Harbin Institute of Technology, where he currently holds the position of an associate researcher. His current research interests include anthropomorphic robot dexterous hands, prosthetics hands, tactile sensors, and the grasping synthesis.

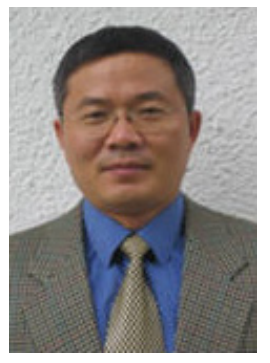

Hong Liu received his Ph.D. degree from Harbin Institute of Technology, Harbin, China, in 1993.

During 1991-1993, he was a joint-train Ph.D. candidate in the Institute of Robotics and System Dynamics, German Aerospace Research Establishment, Wessling, Germany, where, since 1993, he has been a research fellow. His current research interests include the development of dexterous robot hands, space robotics, sensors, and control algorithms. 